Revista Brasileira de Agricultura Irrigada v.11, nº.8, p. 2132 - 2140, 2017

ISSN 1982-7679 (On-line)

Fortaleza, CE, INOVAGRI - http://www.inovagri.org.br

DOI: $10.7127 /$ rbai.v11n800694

Protocolo 694.17 - 06/06/2017 Aprovado em 04/08/2017

\title{
CALIBRAÇÃO DE SONDA TDR PARA A ESTIMATIVA DA UMIDADE EM DIFERENTES TIPOS DE SUBSTRATOS
}

\author{
Rogério de Souza Nóia Júnior ${ }^{1}$, José Eduardo Macedo Pezzopane ${ }^{2}$, Roberto Avelino Cecílio², \\ Bruno Fardim Christo ${ }^{3}$, Jonas Souza Vinco ${ }^{4}$, Talita Miranda Teixeira Xavier ${ }^{5}$
}

\begin{abstract}
RESUMO
Dentre os fatores que contribuem para o uso ineficiente da água na produção agrícola e florestal, destaca-se a dificuldade de se acompanhar a variação temporal e espacial da umidade no solo. Nesse sentido, o TDR (Reflectometria no domínio de tempo) tem se destacado por ser um método de determinação da umidade que provê considerável eficiência, além de ser relativamente fácil de ser manejado. Todavia a eficiência do equipamento na determinação precisa da umidade está diretamente relacionada com a sua calibração para diversos tipos de solos ou substratos. Objetivou-se, ajustar e avaliar equações de calibração para três diferentes tipos de substratos destinados à produção de mudas em viveiros. Os substratos utilizados foram: solo de textura média argilosa (Substrato 1), solo de textura arenosa (Substrato 2) e substrato orgânico (Substrato 3). Os testes mostraram que as equações calibradas para cada tipo de solo foram mais eficientes em determinar a umidade do solo que a equação de Topp et al (1980).
\end{abstract}

Palavras-chaves: Constante dielétrica, Manejo de irrigação e Reflectometria no domínio de tempo.

\section{CALIBRATION OF TDR PROBE FOR ESTIMATING MOISTURE IN DIFFERENT TYPES OF SUBSTRATES}

\footnotetext{
ABSTRACT

Among the factors that contribute to the inefficient use of water in agricultural and forestry production, highlights the difficulty of monitoring the temporal and spatial variation of soil moisture. In this sense, the TDR (Time Domain Reflectometry) has excelled by being a method of determining the moisture that provides considerable efficiency, besides being relatively easy

${ }^{1}$ Mestrando em Engenharia de Sistemas Agrícolas, ESALQ - USP - rogeriosouzanoia@ gmail,com;

2 Professor, Departamento de Ciências Florestais e da Madeira - UFES - pezzopane2007@yahoo.com.br, roberto.cecilio@ufes.br;

${ }^{3}$ Mestre em Produção Vegetal, Centro de Ciências Agrárias e Engenharias - UFES - brunochristo@ @otmail.com;

${ }^{4}$ Engenheiro Florestal, Centro de Ciências Agrárias e Engenharias - UFES - jonasvinco@gmail.com;

${ }^{5}$ Pós doutorando - Departamento de Ciências Florestais e da Madeira, Centro de Ciências Agrárias, Universidade Federal do Espírito Santo - talitamtx@yahoo.com.br.
} 
to use. However, the equipment efficiency in the accurate determination of moisture is directly related to calibration for various types of soils or substrates. The aim of this study was to calibrate a TDR for different types of substrates, as well as to evaluate the performance of different calibration equations. The substrates used were: clay soil (Substrate 1), sandy soil (Substrate 2) and organic substrate (Substrate 3). The tests showed that the adjusted equations for each soil type were superior to Topp et al (1980) equation.

Keywords: Dielectric constant, Irrigation management and Time Domain Reflectometry.

\section{INTRODUÇÃO}

O uso inadequado da água para a produção vegetal é um dos fatores que mais afetam a produtividade agrícola e contribuem para a indisponibilidade hídrica em bacias hidrográficas. Na produção vegetal, quando se utiliza de irrigação, muitas vezes o mau uso da água está associado à dificuldade de se acompanhar a variação temporal e espacial da umidade no solo, a qual é influenciada pelas chuvas, evapotranspiração, drenagem interna, tipo de solo, práticas culturais, dentre outros (SOUZA et al., 2016a).

O monitoramento contínuo da umidade em substratos, usados na produção de mudas em viveiros, também é extremamente importante, uma vez que tanto o excesso quanto a falta de água podem prejudicar o desenvolvimento e crescimento vegetal (ELAIUY et al., 2009). Métodos capazes de determinar o teor de água no solo de forma precisa e instantânea, têm se tornado cada vez mais necessários para obtenção de informações relevantes que devem ser aplicadas ao manejo adequado da irrigação e, consequentemente, dos recursos hídricos.

Diversos métodos são utilizados para a determinação da umidade do solo, destacandose método gravimétrico de estufa, considerado padrão, e os métodos que utilizam da relação entre a umidade e determinadas propriedades físicas dos solos. Neste segundo grupo, destacam-se os sensores ou procedimentos que fazem a determinação de propriedades como o potencial matricial (tensiômetros), a capacidade de atenuação de radiação (raios gama ou sonda de neutrôns) ou as propriedades dielétricas (reflectometria no domínio da frequência - FDR e reflectometria no domínio de tempo - TDR) (CAMARGO et al., 2012).
Dentre os métodos citados, o TDR tem se destacado por ser um método não destrutivo, rápido e que possibilita a obtenção de dados automatizados e contínuos, com custos relativamente baixos (JONES et al., 2002; BIZARI et al., 2011; NARANJO; ATAROFF, 2015). Entretanto, a qualidade dos dados obtidos por estes equipamentos depende diretamente da calibração estabelecida entre a constante dielétrica e a umidade do solo (PONIZOVSKY et al., 1999; SANTOS et al., 2010; ARSOY et al., 2013; CONSOLI et al., 2016).

A técnica do TDR é baseada no efeito da umidade do solo sobre a velocidade de propagação de ondas eletromagnéticas em hastes de sensores inseridas em um meio poroso (UMENYIORA et al., 2012), o qual atua como meio dielétrico. Os materiais dielétricos diferenciam-se quanto à capacidade de ser polarizado por um campo elétrico, o que é representado pela permissividade do meio $(\varepsilon)$, sendo a constante dielétrica $(\mathrm{K})$ o índice que expressa a relação entre a permissividade do meio e a do vácuo.

Sabe-se que o solo é um meio poroso trifásico, constituído pelas partículas sólidas, água e ar. Dentre esses constituintes, a água é o componente com maior influência sobre a constante dielétrica do solo $(\mathrm{K} \cong 81)$, muito maior que as das partículas sólidas orgânicas e minerais ( $K$ entre 2 e 7$)$ e do ar $(K \cong 1)$. Essas diferenças fazem com que a quantidade de água no solo tenha grande influência sobre a constante dielétrica do meio como um todo, denominada constante dielétrica aparente $\left(\mathrm{K}_{\mathrm{a}}\right)$. Desta forma, é possível estimar a umidade volumétrica do meio poroso solo a partir da determinação de seu $\mathrm{K}_{\mathrm{a}}$ (TOPP et al., 1980; WESSOLEK et al., 2009; KAISER et al., 2010). 
Diversos autores têm proposto o uso de modelos matemáticos empíricos que relacionam a constante dielétrica aparente com a umidade (em base volumétrica) dos mais diversos tipos de solo (TOMMASELLI; BACCHI, 2001; NAGARE et al., 2011; SANTOS et al., 2012; SONCELA et al., 2013; SOUZA et al., 2014; ZANETTI et al., 2015), em diferentes meios porosos, como em substratos orgânicos (ELAIUY et al., 2009; VALERO et al., 2009; RAGNI et al., 2012) e em resíduos agroindustriais (SOUZA et al., 2016b). Dentre os modelos matemáticos, a equação polinomial do terceiro grau, proposta por Topp et al., (1980) é uma das mais utilizadas (COMEGNA et al., 2013). Contudo, devido à problemas relacionados aos atributos químicos e físicos de cada meio poroso, a utilização de equipamentos TDR em substratos orgânicos, por exemplo, pode ser ineficiente caso não tenha sido realizado a calibração adequada. Desta forma, o presente trabalho tem o objetivo de ajustar e avaliar equações de calibração para três diferentes tipos de substratos destinados à produção de mudas em viveiros.

\section{MATERIAIS E MÉTODOS}

O estudo foi desenvolvido em casa de vegetação climatizada, com controle de temperatura e umidade relativa do ar, no Laboratório de Meteorologia e Ecofisiologia Florestal da Universidade Federal do Espírito Santo, localizado no município de Jerônimo Monteiro, ES (latitude $20^{\circ} 47^{\prime} 25^{\prime}$ 'S e longitude $41^{\circ} 23^{\prime} 48^{\prime \prime} \mathrm{W}$, altitude de $\left.120 \mathrm{~m}\right)$. Utilizou-se um aparelho TDR Hydrosense II, combinado com a sonda portátil CS 658 (Campbell Scientific, Inc., Logan, UT, USA), em três tipos de substrato: solo de textura média argilosa (Substrato 1, amostrado em Latossolo Vermelho-Amarelo), solo de textura arenosa (Substrato 2, amostrado em Neossolo Quartzarênico), e substrato orgânico (Substrato 3) (Tabela 1). A análise granulométrica dos dois tipos de solo foi realizada em laboratório, de acordo com o método de Embrapa (2011).

Tabela 1. Características descritivas dos três substratos utilizados

\begin{tabular}{ccc}
\hline Substrato & Composição & Densidade $\left(\mathrm{g} \mathrm{cm}^{-3}\right)$ \\
\hline 1 & $43 \%$ de argila, 3\% de silte, 54\% de areia & 1,04 \\
2 & $\begin{array}{c}4 \% \text { de argila, 3\% de silte, 93\% de areia } \\
\text { Mistura homogênea de casca de pinus }\end{array}$ & 1,55 \\
3 & $\begin{array}{c}\text { bioestabilizada, vermiculita, moinha de carvão } \\
\text { vegetal e espuma fenólica }\end{array}$ & 0,43 \\
\hline
\end{tabular}

Utilizaram-se recipientes de plástico de massa conhecida, com dimensões de $21 \mathrm{~cm}$ de profundidade e $20 \mathrm{~cm}$ de diâmetro, preenchidos com os diferentes tipos de substrato. A região inferior dos recipientes foi perfurada para que o excesso de água fosse drenado. Segundo Santos et al. (2010), o tipo de amostra de solo, seja ela desestruturada ou não, não afeta a calibração de sensores do TDR. Desta forma, para os substratos 1 e 2, optou-se pela utilização de amostras deformadas, as quais foram peneiradas em malha $2 \mathrm{~mm}$.

$\mathrm{O}$ estudo foi estabelecido em delineamento inteiramente casualizado, com quatro repetições para cada substrato. Os recipientes completamente preenchidos com os diferentes tipos de substratos foram imersos em água, por um período de 48 horas, para que se tornassem completamente saturados. Posteriormente foram levados para a casa de vegetação, onde as medições foram realizadas. As superfícies superiores dos recipientes foram deixadas expostas, para que pudesse ocorrer livremente o processo de evaporação da água.

A calibração foi realizada a partir de correlação matemática entre as constantes dielétricas aparentes $\left(\mathrm{K}_{\mathrm{a}}\right)$, as quais foram medidas com o uso do TDR, e as umidades de cada substrato (meio poroso), as quais foram determinadas pelo método padrão de estufa (método gravimétrico). 
Diariamente foi realizada a determinação do Ka para o substrato em cada recipiente, utilizando o sensor TDR, e ao mesmo tempo era realizada a pesagem dos recipientes, utilizando balança de precisão. Ao fim do experimento, após a última leitura com o sensor TDR, os substratos foram retirados dos recipientes e levados à estufa a $105^{\circ} \mathrm{C}$, onde permaneceram por 48 horas. Feito isso, foi determinada a umidade volumétrica, através da multiplicação da densidade do substrato pela umidade gravimétrica (obtida pela pesagem) dos substratos pelo método padrão da estufa (valores observados).
A calibração do equipamento para cada meio poroso foi realizada associando-se os valores de umidade volumétrica pelo método padrão de estufa com os valores de constante dielétrica dados pelo equipamento TDR, por meio de ajustes de modelos polinomiais.

\section{Desempenho das equações de estimativa de umidade volumétrica}

Os valores de umidade estimados pela equação proposta por Topp et al. (1980) (Eq. 1) foram comparados com os valores observados pelo método de padrão de estufa, e com as equações de calibração ajustadas para cada substrato estudado.

$$
\theta=-0,053+0,0292 K_{a}-0,000548 K_{a}^{2}+0,000004 K_{a}^{3}
$$

em que:

$\theta$ é a umidade volumétrica, $\mathrm{m}^{3} \mathrm{~m}^{-3}$.

Os resultados foram avaliados utilizando os seguintes indicadores estatísticos: coeficiente de determinação $\left(\mathrm{R}^{2}\right)$, erro médio quadrático (RMSE), índice de concordância ou acurácia (D) (WILLMOTT et al., 1985) e índice de confiança $(C)$, como sugerido por Carmargo e Sentelhas (1997).

$$
\begin{aligned}
& \mathrm{D}=1-\left\{\frac{\sum(\mathrm{Oi}-\mathrm{Pi})^{2}}{\sum(|\mathrm{Pi}-\mathrm{Om}|+|\mathrm{Om}-\mathrm{Pi}|)^{2}}\right\} \\
& C=\mathrm{D} \times \sqrt{\mathrm{R}^{2}}
\end{aligned}
$$

$$
\operatorname{RMSE}=\sqrt{\frac{\sum_{\mathrm{i}=1}^{\mathrm{n}}(\mathrm{Oi}-\mathrm{Pi})^{2}}{\mathrm{n}}}
$$

em que:

Pi é o valor de umidade estimada pelo sensor TDR e pela equação de Topp et al. $(1980)\left(\mathrm{m}^{3} / \mathrm{m}^{3}\right)$;

Oi é valor de umidade encontrada no solo $\left(\mathrm{m}^{3} / \mathrm{m}^{3}\right)$;

Om é a média dos valores de umidade medidos; e

n é o número de eventos.

O desempenho de cada equação de estimativa de umidade a partir da constante dielétrica foi classificado conforme proposto por Camargo e Sentelhas (1997) (Tabela 2).

Tabela 2. Classificação do Coeficiente de Camargo $(C)$ para avaliação de modelos.

\begin{tabular}{cc}
\hline Valores de $C$ & Classificação \\
\hline$>0,85$ & Excelente \\
$0,76-0,85$ & Muito bom \\
$0,66-0,75$ & Bom \\
$0,61-0,65$ & Médio \\
$0,51-0,60$ & Tolerável \\
$0,41-0,50$ & Pobre \\
$\leq 0,40$ & Muito pobre \\
\hline
\end{tabular}

Adaptado de Camargo e Sentelhas (1997). 


\section{RESULTADOS E DISCUSSÃO}

As equações de calibração dos três substratos analisados podem ser observadas na Tabela 3. Verificam-se que os modelos encontrados foram significativos a um grau de confiança superior a 99\% (Prob $>\mathrm{F}$ igual a 0,0001). Os testes estatísticos aplicados ao substrato 1 , indicam que há uma possibilidade alta de o coeficiente cúbico ser nulo (>44\%), o que o torna questionável neste caso (TOMMASELLI; BACCHI, 2001).

Para o substrato 1, adotou-se, portanto, modelo polinomial de segundo grau (Tabela 3). Segundo Tommaselli e Bacchi (2001), em alguns meios porosos, o modelo cúbico tornase menos necessário, o que é o caso do substrato 1. Por outro lado, a equação de calibração dos substratos 2 e 3 , foram ajustadas por meio de um modelo cúbico.

Tabela 3. Resumo da análise de variância das regressões.

\begin{tabular}{cccccccc}
\hline \multirow{2}{*}{$\begin{array}{c}\text { Subs } \\
\cdot\end{array}$} & $\begin{array}{c}\text { Prob> } \\
\mathrm{F}\end{array}$ & $\mathrm{R}^{2}$ aj & Equações & $\begin{array}{c}\text { Termo } \\
\text { independ } \\
\text { ente }\end{array}$ & \multicolumn{3}{c}{ Prob> $\mathrm{T}^{3}$} \\
\hline 1 & 0,000 & 0,95 & $0,055+0,02 k_{a}-0,00038 k_{a}^{2}$ & 0,002 & 0,00 & $\theta^{2}$ & $\theta^{3}$ \\
\hline & 1 & 9 & $-0,000006 k_{a}^{3}$ & & 01 & 0,28 & 0,44 \\
$1^{*}$ & 0.000 & 0.93 & $0,067+0,01699 k_{a}-0,00011 k_{a}^{2}$ & 0,0001 & 0,00 & 0,00 & \\
& 1 & 2 & 01 & 9 & - \\
2 & 0,000 & 0,95 & $0,00016+0,055 k_{a}-0,0045 k_{a}^{2}$ & 0,0001 & 0,00 & 0,00 & 0,00 \\
& 1 & 8 & $-0,00016 k_{a}^{3}$ & 01 & 01 & 01 \\
3 & 0,000 & 0,93 & $0.0662+0,0151 k_{a}-0,00055 k_{a}^{2}$ & 0,0001 & 0,00 & 0,00 & 0,00 \\
& 1 & 8 & $-0,000008 k_{a}^{3}$ & 0,01 & 01 & 01 \\
\hline
\end{tabular}

*Equação polinomial de segundo grau ajustada para o substrato 1.

A dispersão dos pontos em torno das curvas ajustadas (quadrática para o substrato 1 e cúbica para os substratos 2 e 3 ) ocorreu de forma regular em praticamente todos os intervalos de umidade. Contudo, nota-se que as curvas não são coincidentes em quase nenhum momento (Figura 1), o que justifica a calibração do aparelho para cada tipo de solo especificamente.
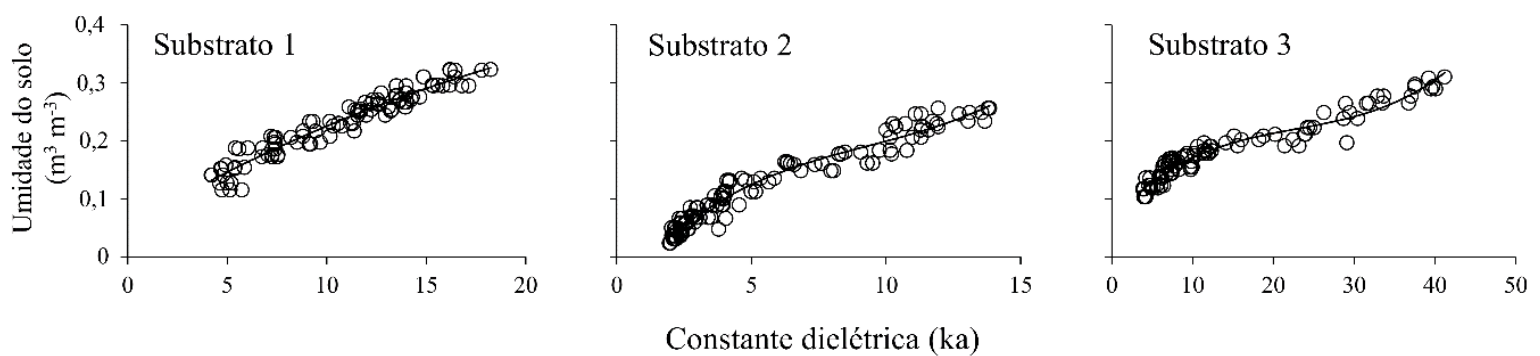

Figura 1. Relações entre a umidade do solo $\left(\mathrm{m}^{3} / \mathrm{m}^{3}\right)$, método padrão de estufa e a constante dielétrica para o substrato 1 (solo de textura média argilosa), substrato 2 (solo de textura arenosa) e substrato 3 (substrato orgânico).

A correlação entre os valores de umidade obtidos pelo método padrão de estufa e pela equação proposta por Topp et al. (1980) e com as equações de calibração podem ser observadas na Figura 2. Verifica-se para a umidade estimada pela equação de Topp et al (1980) que apesar dos coeficientes de determinação $\left(\mathrm{R}^{2}\right)$ serem altos $(>0.91)$, os coeficientes angulares das equações são diferentes de um, e os coeficientes lineares diferentes de zero, indicando desempenho ruim desta equação. A baixa eficiência da equação, torna-se ainda mais pronunciada no substrato 3, principalmente quando a umidade 
estimada era superior que $0.15 \mathrm{~m}^{3}$ $\mathrm{m}^{-3}$, onde houve tendência em superestimar os valores de umidade.
Quando a umidade do solo era inferior a $0.15 \mathrm{~m}^{3} \mathrm{~m}^{-3}$, a tendência foi de subestimar os valores.
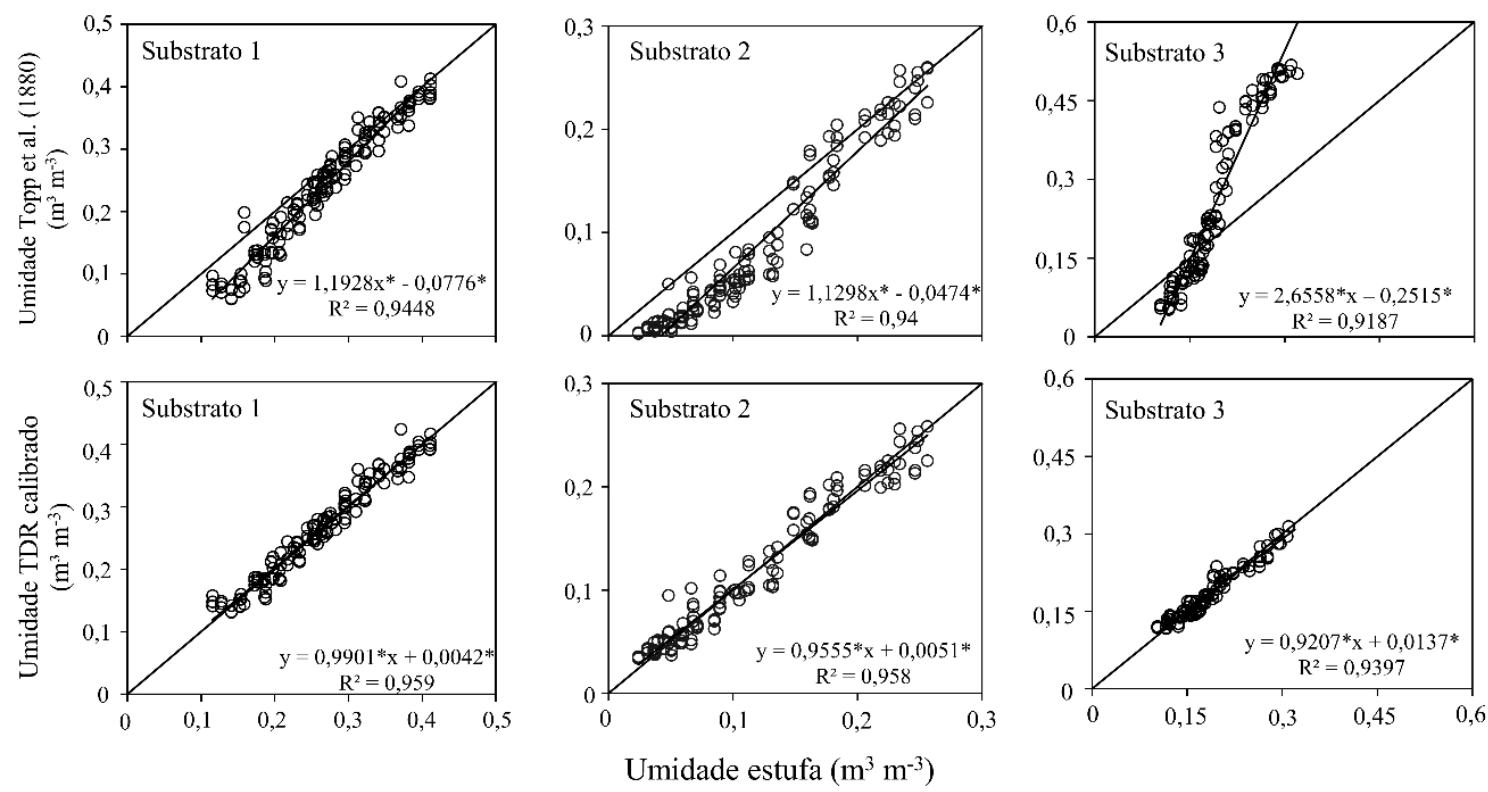

Figura 2. Correlação entre a umidade do solo via método padrão de estufa e umidade do solo via TDR em diferentes tipos de substratos.

*Significativo a $5 \%$ de probabilidade

Nota-se, que com a calibração do equipamento, os coeficientes angulares se aproximaram de um e os lineares de zero, indicando que com a calibração adequada, o equipamento TDR se torna eficiente na determinação da umidade do solo.

$\mathrm{Na}$ tabela 4, apresentam-se os valores do coeficiente de determinação $\left(\mathrm{R}^{2}\right)$, a raiz do erro quadrático médio (RMSE), o índice de concordância (D) e o índice de confiança $(C)$ para todas equações e em todos os substratos analisados. Verifica-se nesta tabela que a equação proposta por Topp et al (1980) apresenta índices de confiança classificados como excelentes nos substratos 1 e 2 . Todavia, a equação foi classificada como bom no substrato 3, tendo apresentado os maiores valores de RMSE neste meio poroso.

Tabela 4. Coeficiente de determinação $\left(\mathrm{R}^{2}\right)$, erro médio quadrático (RMSE), índice de concordância ou acurácia (D) e índice de confiança $(C)$ das equações analisadas neste estudo.

\begin{tabular}{cccccc}
\hline Equações & $\mathrm{R}^{2}$ & RMSE & $\mathrm{D}$ & $\mathrm{C}$ & $\begin{array}{c}\text { Classificação do } \\
\text { coeficiente de Camargo } \\
\text { (C) }\end{array}$ \\
\hline \multicolumn{7}{c}{$\begin{array}{c}\text { Substrato 1 } \\
\text { Topp et al. (1980) }\end{array}$} & 0,94 & 0,0436 & 0,94 & 0,91 & Excelente \\
Calibrado & 0,96 & 0,0154 & 0,99 & 0,97 & Excelente \\
\hline \multicolumn{7}{c}{ Substrato 2 } \\
\hline Topp et al. (1980) & 0,94 & 0,0385 & 0,93 & 0,90 & Excelente \\
Calibrado & 0,96 & 0,0141 & 0,97 & 0,95 & Excelente \\
\hline \multicolumn{7}{c}{ Substrato 3 } \\
\hline Topp et al. (1980) & 0,91 & 0,1182 & 0,70 & 0,67 & Bom \\
Calibrado & 0,94 & 0,0005 & 0,95 & 0,93 & Excelente \\
\hline
\end{tabular}


O fato do maior RMSE ter sido encontrado no substrato 3 , pode ser explicado pela alta presença de matéria orgânica no substrato, o que segundo diversos autores interfere na leitura da constante dielétrica realizada pelo equipamento (SCHAAP et al., 1997; PERSSON; UVO, 2002; GHEZZEHEI, 2008). Diversos autores relatam a necessidade de se estabelecer relações específicas entre $\mathrm{Ka}$ e a umidade do solo para meios porosos com altos teores de matéria orgânica (Ponizovsky et al., 1999; Gonçalves et al, 2011). Kameyama e Miyamoto (2008) indicam que a microestrutura dos solos influencia os valores de Ka. No caso da matéria orgânica, características como a textura, densidade do substrato, área superficial específica, calor específico e consequentemente, a variação da temperatura do solo ao longo do perfil no momento da leitura podem afetar a medida de $\mathrm{Ka}$, prejudicando assim a estimativa da umidade do solo (Gonçalves et al, 2011).

De forma geral, a calibração demonstrou ser muito importante, uma vez que reduziu os valores de RMSE. Por outro lado, nota-se que embora o modelo de Topp et al. (1980) tenha sido eficaz na determinação da umidade do solo dos substratos 1 e 2, a mesma apresentou rendimento inferior no substrato 3 o que a torna não aplicável para determinação de umidade neste meio poroso.

\section{CONCLUSÃO}

As curvas de calibração obtidas em cada solo individualmente apresentaram boa eficácia, uma vez que, apresentaram os menores erros, melhor exatidão e precisão.

O modelo de Topp et al. (1980) demonstrou ser muito eficiente, dentro de determinadas faixas, para solos com texturas média argilosa e arenosos. Porém para o substrato com alta presença de matéria orgânica, o modelo não apresentou eficácia.

\section{REFERÊNCIAS}

ARSOY, S.; OZGUR, M.; KESKIN, E.; YILMAZ, C. Enhancing TDR based water content measurements by ANN in sandy soils.

Geoderma, v. 195, p. 133-144, 2013.

BIZARI, D. R.; MATSURA, E. E.; SOUZA, C. F.; ROQUE, M. W. Haste portátil para utilização de sondas de TDR em ensaios de campo. Irriga, v. 16, n. 1, p. 31-41, 2011.

CAMARGO A. P.; SENTELHAS P. C. Performance of different potential evapotranspiration methods in the state of São Paulo, Brazil. Revista Brasileira Agrometeorologia, v. 5, p. 89-97, 1997.

CAMARGO, D. C.; TENDERO, J. I. C.; ÁlvarEZ, J. F. O.; SEVILLA, F. M. Calibração da sonda de capacitância ENVIROSCAN®. Irriga, Edição Especial, p. 27 - 39, 2012.

COMEGNA, A.; COPPOLA, A.; DRAGONETTI, G.; SEVERINO, G.; SOMMELLA, A.; BASILE, A. Dielectric properties of a tilled sandy volcanic-vesuvian soil with moderate andic features. Soil and Tillage Research, v. 133, p. 93-100, 2013.

CONSOLI, S.; LICCIARDELLO, F.; VANELLA, D.; PASOTTI, L.; VILLANI, G.; TOMEI, F. Testing the water balance model criteria using TDR measurements, micrometeorological data and satellite-based information. Agricultural Water Management, v. 170, p. 68-80, 2016.

EMBRAPA, 2011. Manual de Métodos de Análise de Solo, $2^{\circ}$ Edição revista, Rio de Janeiro, p. 230.

ELAIUY, C. L. M.; SATO, M. L.; VARALLO, A. C. T.; SOUZA, C. F. Desenvolvimento e avaliação de sonda de TDR para o manejo racional da água em substratos utilizados na produção de mudas florestais. Revista Ambiente \& Água, v.4, n.1, 2009. 
GHEZZEHEI, T. A. Errors in determination of soil water content using time domain reflectometry caused by soil compaction around waveguides. Water Resources Research, v. 44, 2008.

GONÇALVES, A. C. A.; TRINTINALHA, M. A.; TORMENA, C. A.; FOLEGATTI, M. V. Influência da densidade do solo na estimativa da umidade em um Nitossolo Vermelho Distroférrico, por meio da técnica de TDR. Revista Brasileira de Ciência do Solo, v. 35, p. 1551- 1559, 2011.

JONES, B. S.; WRAITH, J. M.; OR, D. Time domain reflectometry measurement principles and applications. Hydrological Processes. V. 16, p. 141-153, 2002.

KAISER， D. R.; REINERT， D. J.; REICHERT, J. M.; MINELLA, J. P. G. Dielectric constant obtained from TDR and volumetric moisture of soils in southern Brazil. Revista Brasileira de Ciência do Solo, v. 34, p. 649-658, 2010.

KAMEYAMA, K.; MIYAMOTO, T. Measurement of solid phase permittivity for volcanic soils by time domain reflectometry. European Journal of Soil Science, v. 59, p. 1253-1259, 2008.

NAGARE, R. M.; SCHINCARIOL, R. A.; QUINTON, W. L.; HAYASHI, M. Laboratory calibration of time domain reflectometry to determine moisture content in undisturbed peat samples. European Journal of Soil Science, v. 62, p. 505-515, 2011.

NARANJO, M. R.; ATAROFF, M. Calibración de equipos TDR su usos en suelos non disturbados. Asociación Interciencia, v. 40, n. 6, p. 416-422, 2015.

PERSSON, M.; UVO, B. C. Estimating soil solution electrical conductivity from time domain reflectometry measurements using neural networks. Journal of Hydrology, v. 273, n. 1, p. 249-256, 2002.
PONIZOVSKY, A. A.; CHUDINOVA, S. M.; PACHEPSKY, Y. A. Performance of TDR calibration models as affected by soil texture. Journal of Hydrology, v. 218, n. 1, p. 35-43, 1999.

SANTOS, B. S.; CECÍLIO, R. A.; COELHO, E. F.; BATISTA, R. O.; SILVA, J. P. S. Calibração de TDR: desempenho de alguns métodos e equações de calibração. Revista Agrarian, v.5, n.16, p.131-139, 2012.

RAGNI, L.; BERARDINELLI, A.; CEVOLI, C.; VALLI, E. Assessment of the water content in extra virgin olive oils by Time Domain Reflectometry (TDR) and Partial Least Squares (PLS) regression methods. Journal of Food Engineering, v. 111, n. 1, p. 66-72, 2012.

SANTOS, M. R.; ZONTA, J. H.; MARTINEZ, M. A. Influência do tipo de amostragem na constante dielétrica do solo e na calibração de sondas de TDR. Revista Brasileira de Ciência do Solo, v. 34, n. 2, p. 299-308, 2010.

SCHAAP, M. G.; DE LANGE, L.; HEIMOVAARA, T. J. TDR calibration of organic forest floor media. Soil Technology, v.11, n.2, p.205-217, 1997.

SONCELA, R.; SAMPAIO, S. C.; VILAS BOAS, M. A.; TAVARES, M. H. F.; SMANHOTTO, A. Construction and calibration of TDR probes for volumetric water content estimation in a Distroferric Red Latosol. Engenharia Agrícola, v. 33, n. 5, p. 919-928, 2013.

SOUZA, C. F.; CIRILO, L. S.; BASTOS, R. G.; PERES, J. G.; OLIVEIRA, A. O.; Sonda de tdr para a estimativa de umidade em bagaço de cana-de-açúcar. Engenharia agrícola, v.36, n.1, p.24-35, 2016b.

SOUZA, C. F.; PIRES, R. C.; DE MIRANDA, D. B.; VARALLO, A. C. Calibração de sonda FDR e TDR para a estimativa da umidade em dois tipos de solo. Irriga, v. 18, n. 4, p. 597606, 2014. 
SOUZA, C. F.; SILVA, C. R.; ANDRADE JÚNIOR, A. S.; COELHO, E. F. Monitoramento do teor de água no solo em tempo real com as técnicas de TDR e FDR. Irriga, Edição Especial, p. 26-42, 2016a.

TOMMASELLI, J. T. G; BACCHI, O. O. S. Calibração de um equipamento de TDR para medida da umidade de solos. Pesquisa Agropecuária Brasileira. v. 36, p. 11451154, 2001.

TOPP, G. C.; DAVIS, J. L.; ANNAN, A. P. Eletromagnetic determination of soil water content: measurements in coaxial transmission lines. Water Resources Research. v. 16, p. 574-582, 1980.

UMENYIORA, C. A.; DRUCE, R. L.; CURRY, R. D.; NORGARD, P.; MCKEE, T.; BOWDERS, J. J.; BRYAN, D. A. Dielectric constant of sand using TDR and FDR measurements and prediction models (timedomain reflectometry)(frequency-domain reflectometry)(Technical report). IEEE Transactions on Plasma Science, v. 40, n. 10, p. 2408-2416, 2012
VALERO, R. M. M.; MATSURA, E. E.; SOUZA, A. L. Caracterização física de dois substratos orgânicos para plantas e a estimativa da umidade por meio da reflectometria no domínio do tempo. Ciência Rural, v.39, n.2, p.571-574, 2009.

WESSOLEK, G.; STOFFREGEN, H.; TAUMER, K. Persistency of flow patterns in a water repellent sandy soil - Conclusions of TDR readings and a time-delayed double tracer experiment. Journal of Hydrology, $\mathrm{v}$. 375, n. 3-4, p. 524-535, 2009.

WILLMOTT, C. J.; ACKLESSON, S. G.; DAVIS, R. E.; FEDDEMA, J. J.; KLINK, K. M.; LEGATES, D. R.; O'DONNELL, J.; ROWE, C. M. Statistics for the evaluation and comparison of models. Journal of Geophysical Research, v. 90, p. 8995-9005, 1985.

ZANETTI, S. S.; CECÍLIO, R. A.; SILVA, V. H.; ALVES, E. G. General calibration of TDR to assess the moisture of tropical soils using artificial neural networks. Journal of Hydrology, v. 530, p. 657-666, 2015. 